Chaotic behavior and fractals discovered in the time evolution of discharge current at atmospheric pressure

T. Fukuyama, K. Ota, and H. Sakamoto

Citation: Physics of Plasmas 25, 092303 (2018); doi: 10.1063/1.5042615

View online: https://doi.org/10.1063/1.5042615

View Table of Contents: http://aip.scitation.org/toc/php/25/9

Published by the American Institute of Physics

\section{PHYSICS TODAY}

WHITEPAPERS
MANAGER'S GUIDE

Accelerate R\&D with Multiphysics Simulation
READ NOW

PRESENTED BY $\checkmark \subset \bigcirc M S O L$ 


\title{
Chaotic behavior and fractals discovered in the time evolution of discharge current at atmospheric pressure
}

\author{
T. Fukuyama, K. Ota, and H. Sakamoto \\ Faculty of Education, Nagasaki University, 1-14 Bunkyo-machi, Nagasaki 852-8521, Japan
}

(Received 3 June 2018; accepted 29 August 2018; published online 17 September 2018)

\begin{abstract}
In this study, nonlinear behaviors in the time evolution of discharge current at atmospheric pressure are investigated from the viewpoints of chaos and fractals. When the electric field between two electrodes placed in atmospheric pressure increases and exceeds a certain value, the insulation breakdown of air and corona discharge occur. With the further increase in the electric field, the corona discharge gradually transforms into an arc discharge along with the increasing discharge current. It is found from the chaotic analysis results of the calculation of the largest Lyapunov exponents that the system sustains more chaos in the corona than the arc discharge, i.e., the system is stabilized with the system changing from a corona to arc discharge. The correlation dimension of the time evolution of the discharge current saturates at a non-integer value at every discharge current, and $\frac{1}{f}$-type spectra are observed in the power spectrum, i.e., the system exhibits fractal properties. Published by AIP Publishing. https://doi.org/10.1063/1.5042615
\end{abstract}

\section{INTRODUCTION}

For several decades, nonlinear phenomena such as chaos and fractals have attracted significant attention globally in all fields of science, and their concepts are considered common and beneficial according to the investigation of the structures caused by discharge phenomena. Many different varieties of experimental studies on nonlinear behaviors have been accomplished in plasmas produced by discharges, ${ }^{1-6}$ observations of chaos, ${ }^{7-19}$ control of chaos, ${ }^{20-22}$ synchronization of coupled oscillators, ${ }^{23-28}$ and calculations of fractal dimensions. $^{29-35}$ The properties concerning chaos and fractals can be characterized quantitatively, for example, from experimental data using the calculation of the Lyapunov exponent $^{36}$ and the correlation dimension, ${ }^{37}$ respectively.

As mentioned above, many types of nonlinear behavior, such as chaos and fractals, have been investigated in plasma. The nonlinear system that presents chaotic behaviors also possesses the properties of fractals at the same time. Corona and arc discharge at atmospheric pressure exhibit complex behaviors temporally and spatially, caused by the instability of electron streams because of insulation breakdown in air. To our knowledge, no study on the dynamic behaviors of discharge at atmospheric pressure from the viewpoint of both chaos and fractals has been reported. It is expected that understanding the basic properties of nonlinear discharge at atmospheric pressure can be useful in a wide range of applications of plasma produced by discharge. For these reasons, a study on the nonlinear behaviors of discharge at atmospheric pressure is performed from the viewpoint of chaos and fractals.

There are several types of discharges, e.g., corona, ${ }^{38-41}$ arc, $^{42-44}$ glow, ${ }^{45,46}$ and dielectric barrier, ${ }^{4-51}$ depending on their experimental conditions such as pressure, discharge current, configuration, and shape of electrode. In a situation where two electrodes are placed such that they maintain a certain distance in atmospheric pressure, when the strength of the electric field exceeds a certain value, i.e., threshold, the insulation breakdown of atmospheric air, and corona discharge occur. ${ }^{38-41}$ With the increase in the electric field between the electrodes, the corona discharge gradually changes shape into an arc discharge as a result of the increasing discharge current.

In this study, we focus on the time evolution of the fluctuations in the discharge current observed in the phenomenon of a corona discharge transforming to an arc discharge at atmospheric pressure from the viewpoint of chaos and fractals. This paper is organized as follows. The experimental setup and configuration are described in Sec. II. The analysis of the time series and power spectra of the system transforming from a corona to arc with discharge current is reported in Sec. III. The results and discussion concerning the properties of the chaos and fractals discovered in the time evolution of the discharge system, by calculating the Lyapunov exponent and the correlation dimension, are shown in Sec. IV. Section IV presents the key portion of the argument in this paper, and finally, the findings are summarized in Sec. V.

\section{EXPERIMENTAL SETUP AND CONFIGURATION}

A schematic of the experimental setup used to investigate the dynamics in the time evolution of the discharge current at atmospheric pressure is shown in Fig. 1. Two stainless-steel electrodes of nails with a diameter and length of 4.3 and $45 \mathrm{~mm}$, respectively, are arranged at a fixed distance from each other of $5.7 \mathrm{~mm}$. All the experiments were performed under atmospheric pressure. Time series signals for the analysis are obtained as fluctuations in the intensity of the discharge current using a clamp on a probe (3276, HIOKI) with a power supply (3272, HIOKI) and recorded using a digital oscilloscope (GDS-1072A-U, GWINSTEK). Here, it should be noted that the time series signals are sampled by the horizontal extension around the central position of the oscillations in the discharge current. A high-speed 


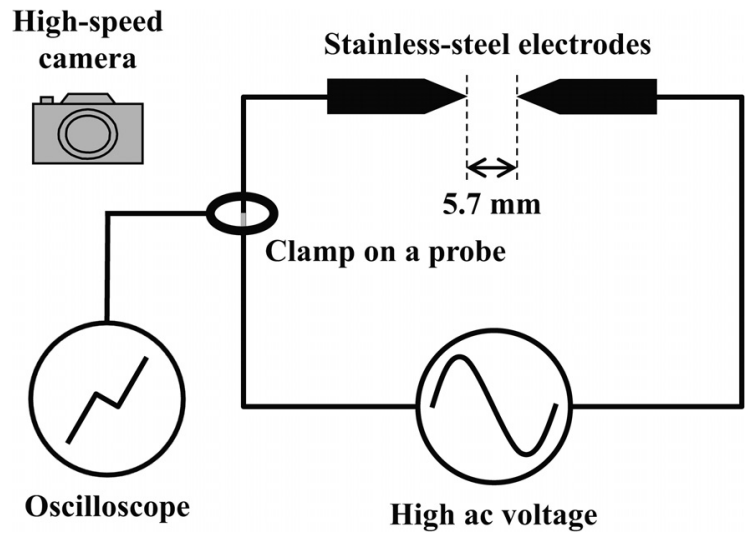

FIG. 1. Schematic of the experimental setup. Two stainless-steel electrodes of nails with a diameter and length of 4.3 and $45 \mathrm{~mm}$, respectively, are arranged a certain distance apart: $5.7 \mathrm{~mm}$. Experiments are performed under atmospheric pressure. Time series signals for the analysis are obtained as fluctuations in the intensity of the discharge current using a clamp on a probe and recorded using a digital oscilloscope. A high-speed camera is used for the measurements. A strong ac electric field is generated using a versatile variable ac outlet and neon sign transformer and applied horizontally between the electrodes. The ac frequency is $60 \mathrm{~Hz}$.

camera (EX-F1, CASIO) with a lens (focus: $7.3-87.6 \mathrm{~mm}$ ) is used for the measurements. High ac voltages, applied between the electrodes for discharge, are generated using a versatile variable ac outlet and a neon sign transformer (L704143G, LECIP). In this manner, a strong ac electric field is applied horizontally between the electrodes. The frequency of the ac was $60 \mathrm{~Hz}$.

The dynamics versus discharge voltage are as follows: corona discharge occurs with the accompanying insulation breakdown of air when the electric field between the electrodes becomes more than about $7000 \mathrm{~V}$, and with the increase in discharge current, the discharge state gradually transforms to an arc discharge at about $10000 \mathrm{~V}$; there exists no clear threshold between both discharge states in this experiment. For example, in the case of a vacuum discharge tube, it should be noted that the applied voltage between electrodes usually decreases drastically with discharge current immediately after the system has changed from a glow to arc discharge. ${ }^{52}$ However, in this experiment, such voltage depression does not occur, because the applied voltage is kept constant using a constant-voltage source.

\section{ANALYSIS OF TRANSITIONS FROM CORONA TO ARC DISCHARGE}

As described before, the corona discharge changes its form to an arc discharge with the increase in discharge current, i.e., increasing electric field, which flows between the two electrodes. When the electric field exceeds about $7000 \mathrm{~V}$, corona discharge occurs with the accompanying insulation breakdown of air. Corona discharge is an unstable and imperceptible discharge, and it occurs under atmospheric pressure and a significantly non-uniform electric field around the electrode. When the electric field is $7179 \mathrm{~V}$ (typical corona discharge), the time evolutions have $\frac{1}{f}$ spectra that exhibit chaotic oscillations, which have repeating laminar and burst spikes, as shown in Fig. 2. Figures 2(a)-2(c) present the time series signals of the discharge current, power spectrum constructed from (a), and an image captured by the high-speed camera, respectively. Figure 2(a) indicates that the oscillation of $60 \mathrm{~Hz}$ caused by the ac discharge source is interrupted by strong and frequent spikes. It can be seen from Fig. 2(b) that there exist $\frac{1}{f}$ fluctuations in the lowfrequency region. The time series exhibits chaotic oscillations, and the power spectrum shows one of the typical properties of fractals. ${ }^{53}$ Figure 2(c) shows the state of the corona discharge, where a number of fine discharge filaments with light emission can be observed. Correlation dimension is around 1.5. In the case of corona discharge, the discharge occurs under a significantly non-uniform electric field around the electrodes. Therefore, the corona discharge has a filamentary shape due to the instability caused by insulation breakdown. Furthermore, corona discharge occurs accompanied by a violent sound of intermittent breakdown.

When the electric field increases further, the discharge state gradually transforms from a corona to an arc along with (a)

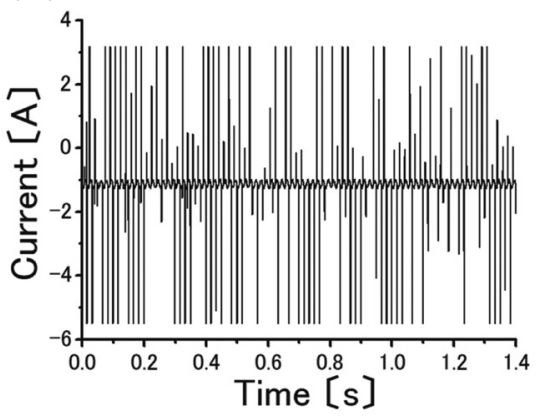

(b)

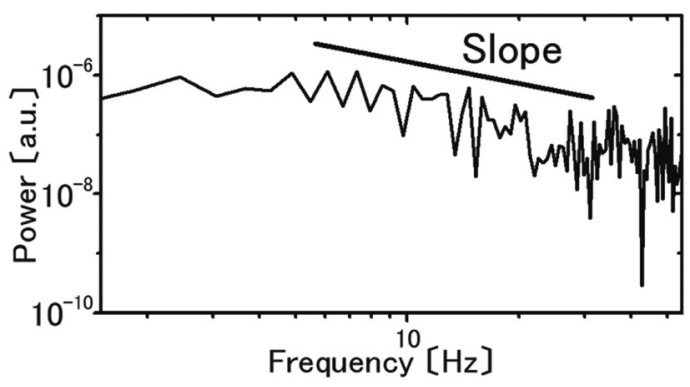

FIG. 2. (a) Time series signals of discharge current, (b) power spectrum constructed from (a), and (c) image of corona discharge captured by highspeed camera. Correlation dimension is around 1.5 .

(c)

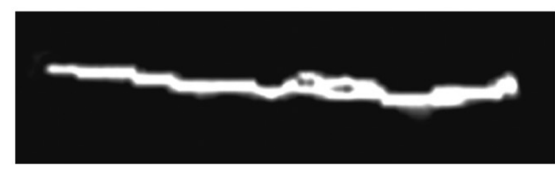


(a)

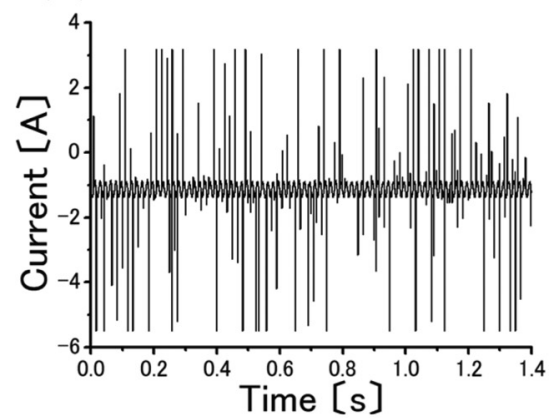

(b)

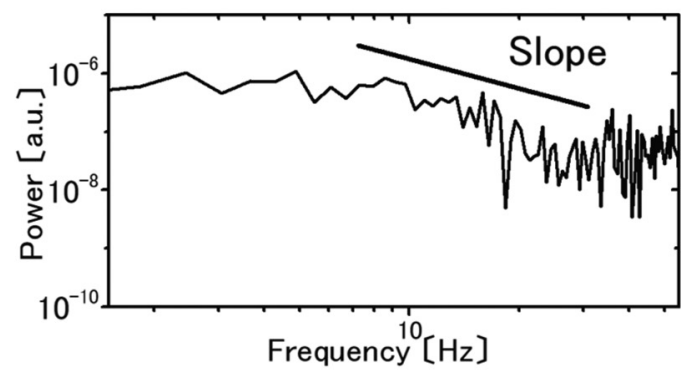

FIG. 3. (a) Time series signals of discharge current, (b) power spectrum constructed from (a), and (c) image of arc discharge captured by a high-speed camera. Correlation dimension is around 1.35. The arc spots are indicated by the two arrows.

(c)

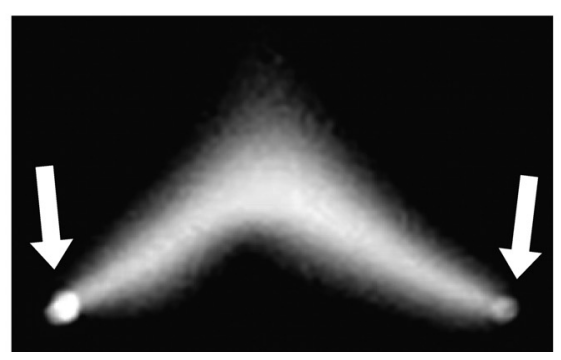

the increasing discharge current. The arc discharge is a stable and perceptible discharge compared to the corona discharge. In the arc discharge, ions produced by ionization collide with the electrodes, which then emit electrons; as a result, the atmospheric air is highly ionized compared with the case of the corona discharge. When the electric field is $13139 \mathrm{~V}$ (typical arc discharge), the time evolutions exhibit $\frac{1}{f}$ spectra, which have repeating laminar and burst spikes, as shown in Fig. 3. Figure 3 is roughly similar to Fig. 2; however, the amplitude of oscillation in the laminar state increases and the number of burst spikes decreases. Figures 3(a)-3(c) present the time series signals of the discharge current, power spectrum constructed from (a), and an image captured by the high-speed camera, respectively. Figure 3(c) shows the state of the arc discharge, where a bright arc spot is observed at the tip of the nail corresponding to the root of the arc discharge. Correlation dimension is around 1.35. The arc spots are indicated by the two arrows. Such a bright spot appears when the discharge system changes from corona to arc discharge. The arc plasma assumes an upward convex shape. The cause of this phenomenon is similar to that which causes the behavior of the central part of the arc plasma filament in the pathway of the discharge that is lifted because of the vertical updraft of heat. In the case of arc discharge, the discharge continues stably, compared with corona discharge, according to the increasing discharge current. Sufficient discharge current flows between the two electrodes; therefore, the pathway of the arc discharge is sufficiently ionized, stabilized, and diffused. Furthermore, arc discharge is more silent compared with corona discharge.

The time series of the discharge current, sampled using a clamp on a probe, is analyzed. Figures 4(a) and 4(b) show the average numbers of spikes and average intensity of the discharge current, respectively, over a time interval with the (a)

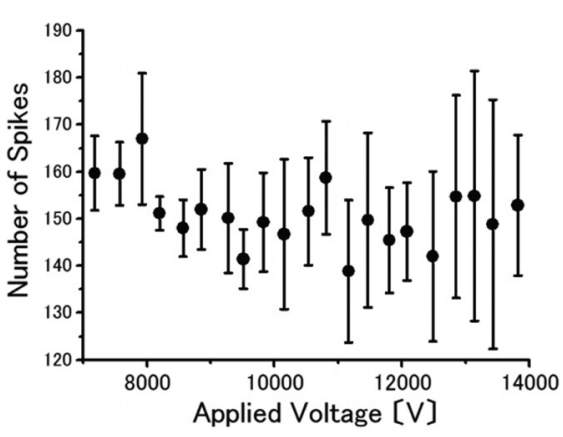

(b)

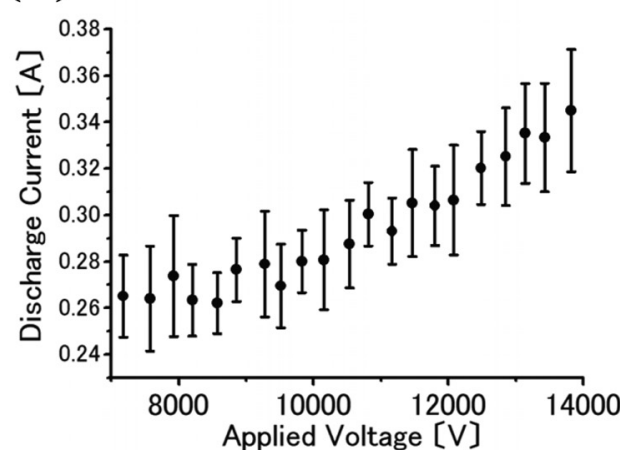

FIG. 4. (a) Average numbers of spikes and (b) average intensity of discharge current over a time interval with the applied voltage between the electrodes. The error bars have been calculated using the standard deviation of multiple measurements. 
(a)

(b)
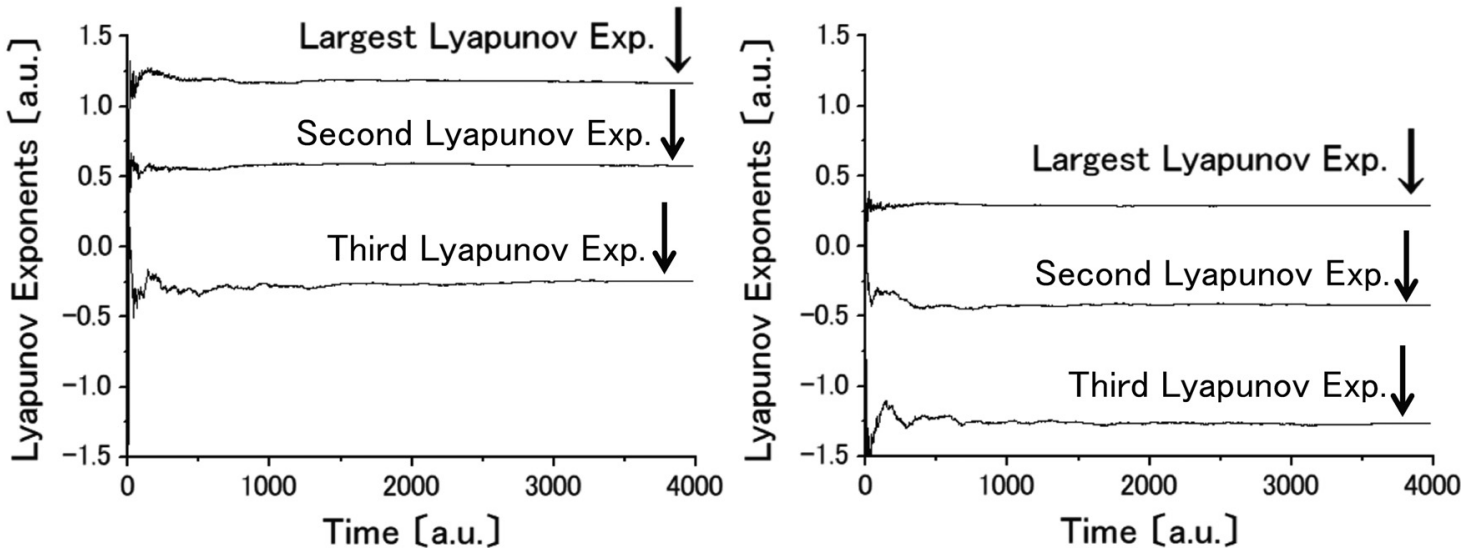

FIG. 5. Lyapunov exponents calculated from time series corresponding to (a) 7179 and (b) $13139 \mathrm{~V}$ (typical corona and arc discharge values, respectively) shown in Figs. 2 and 3.

applied voltage between the electrodes. The error bars have been calculated using the standard deviation of multiple measurements. According to the transition from corona to arc discharge and from the time series shown in Figs. 2(a) and 3(a), it seems that the average number of spikes decreases drastically. However, many small spikes exist in the arc discharge; therefore, the average number of spikes does not decrease drastically. Next, the average intensity of discharge current with the applied voltage is calculated from each time series graph, as shown in Fig. 4(b). From this figure, the analysis shows that the average intensity of the discharge current increases in almost direct proportion to the applied voltage.

\section{CHAOS AND FRACTALS DISCOVERED IN TIME EVOLUTION OF THE DISCHARGE SYSTEM}

As explained above, the time evolution of the discharge current at atmospheric pressure sustains chaotic oscillations, and parts of the time series simultaneously have a selfsimilarity to other parts of the time series, i.e., fractal-like property. In this section, such properties are investigated by quantitative analysis concerning chaos and fractals using a time series obtained in a string of experiments.

From the viewpoint of the complexity of the time evolution, such as chaotic behavior, of discharge current with the applied voltage, the Lyapunov exponents of the time series are calculated to perform chaotic analysis to quantitatively examine the complexity, based on the algorithm reported in Ref. 36. Figure 5 shows the Lyapunov exponents calculated from the time series corresponding to (a) 7179 and (b) $13139 \mathrm{~V}$ (typical corona and arc discharge values, respectively) shown in Figs. 2 and 3. Focusing on the largest Lyapunov exponent, it has a higher value in the case of $7179 \mathrm{~V}$ (corona discharge) than $13139 \mathrm{~V}$ (arc discharge). The value of the largest Lyapunov exponent is positive for chaotic oscillations; this value is higher for more chaos, whereas it becomes close to zero for the system with periodic oscillations.

Figure 6 presents a graph of the largest Lyapunov exponents for discharge voltage. The error bars of each plot have been calculated using the standard deviation of multiple measurements. From this figure, the analysis indicates that the largest Lyapunov exponents decrease with the applied voltage, that is, the system is gradually stabilized with increasing applied voltage, i.e., discharge current intensity. In the corona discharge state, the time series exhibits many spikes intricately caused by the insulation breakdown of air. In contrast, in the arc discharge state, the discharge system becomes stabilized with the increase in the flow of current. From these reasons, the phenomenon shown in Fig. 6 can be understood.

From the viewpoint of the self-similarity of the time evolution, such as fractals, of the discharge current for the applied voltage, the correlation dimensions of the trajectories constructed in phase space from the time series are calculated to perform chaotic analysis to quantitatively examine the self-similarity, based on the algorithm reported in Ref. 37. The so-called "correlation dimension" is appropriately used to estimate the dimensions of the trajectories. The

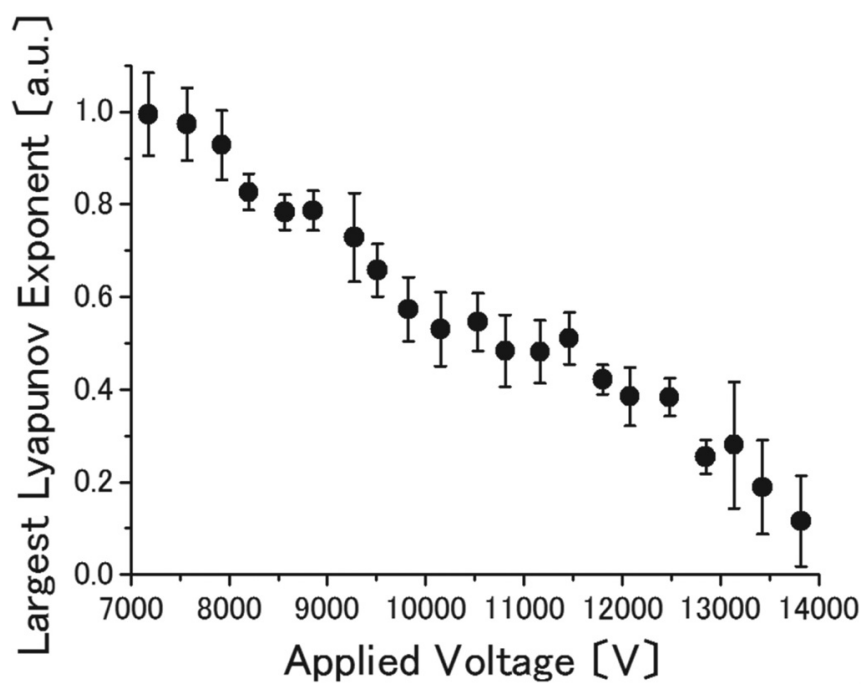

FIG. 6. Graph of the largest Lyapunov exponents for discharge voltage. The error bars of each plot have been calculated using the standard deviation of multiple measurements. 
(a)

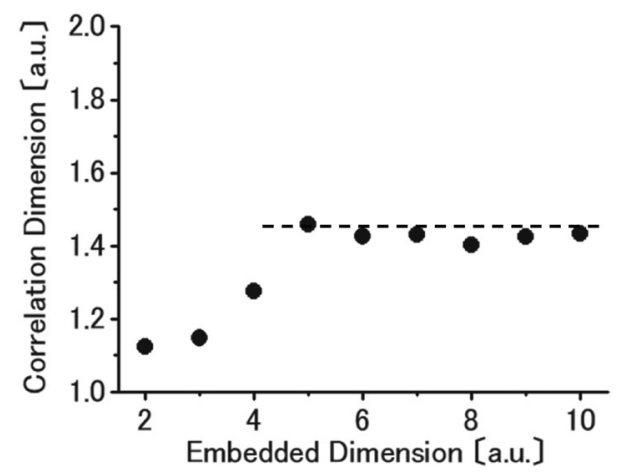

(b)

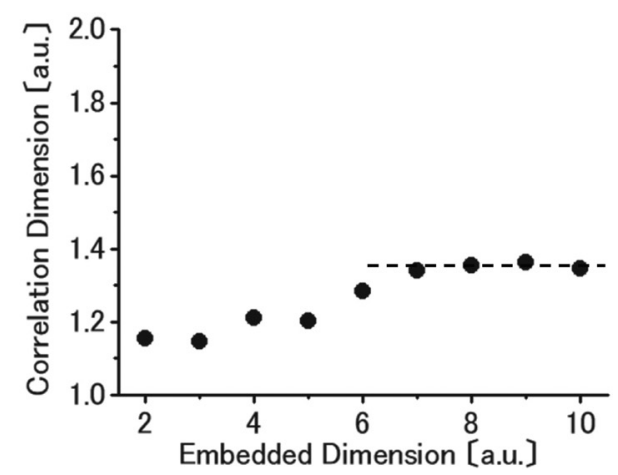

FIG. 7. Correlation dimensions calculated, with varying embedded dimensions, from time series corresponding to (a) 7179 and (b) $13139 \mathrm{~V}$ (typical corona and arc discharge values, respectively) shown in Figs. 2 and 3. various forms of the dimensions of the trajectories and ways of calculating them analytically and numerically are wellexplored subjects. One of the key developments can be found in Grassberger and Procaccia. ${ }^{37}$ The correlation dimension $D$ can be calculated according to the following procedure:

$$
D=\lim _{\varepsilon \rightarrow 0} \lim _{N \rightarrow \infty} \frac{\ln C(\varepsilon, N)}{\ln \varepsilon},
$$

where $C(\varepsilon, N)$ is the correlation integral, $\varepsilon$ is the size of a partition element of phase space, and $N$ is the number of points used to estimate the dimension. The correlation integral $C(\varepsilon, N)$ can be calculated as follows:

$$
C(\varepsilon, N)=\frac{1}{N^{2}} \sum_{\substack{i, j=1 \\ i \neq j}}^{N} H\left(\varepsilon-\left|\mathbf{X}_{\mathbf{i}}-\mathbf{X}_{\mathbf{j}}\right|\right),
$$

where $H$ is the Heaviside function and $\mathbf{X}$ is a phase vector.

One can numerically estimate the correlation dimension $D$ as the slope of the best-fit straight line to a numerical plot of $\ln C(\varepsilon, N)$ versus $\ln \varepsilon$. In addition, the dependence of $D$ on the embedded dimension can be analyzed. If the dimension of the original trajectories is finite, as the embedded dimension increases, the value of $D$ saturates. Here, the correlation dimension saturates at a non-integer value for the chaotic trajectory, whereas it saturates at an integer value for the periodic trajectory.

Figure 7 shows the correlation dimensions calculated, with varying embedded dimensions, from the time series corresponding to (a) 7179 and (b) $13139 \mathrm{~V}$ (typical corona and arc discharge values, respectively) shown in Figs. 2 and 3. As described above, the correlation dimension $D$ can be numerically estimated as the slope of the best-fit straight line to a numerical plot of $\ln C(\varepsilon, N)$ versus $\ln \varepsilon$. Focusing on the correlation dimension, both saturate at a non-integer value: 1.5 (corona discharge) and 1.35 (arc discharge). These values are higher in the case of $7179 \mathrm{~V}$ (corona discharge) than $13139 \mathrm{~V}$ (arc discharge). The correlation dimension has a non-integer value for the fractal. This value becomes closer to the integer value for the system with periodic oscillations.

Figure 8 presents a graph of the correlation dimensions for the discharge voltage. The error bars of each plot have been calculated using the standard deviation of multiple measurements. From this figure, the analysis indicates that the system exhibits a correlation dimension of about 1.5 in the corona discharge, which then gradually decreases to about 1.35 with the applied voltage. Throughout the transformation from corona to arc discharge, the time series with the laminar oscillation state is interrupted by many spikes. Despite the decrease in the number of spikes with discharge current, the self-similar property in the time series is maintained. Throughout the discharge in automorphic air, the correlation dimension of the time series of the discharge indicates the non-integer value between 1.0 and 2.0; therefore, the system has the freedom of low dimension.

Figures 9(a) and 9(b) present graphs of the largest Lyapunov exponents and the correlation dimensions, respectively, for the average intensity of the discharge current, obtained in Fig. 4(b). The error bars of each plot have been calculated using the standard deviation of multiple measurements. From this figure, the following can be concluded: The largest Lyapunov exponent is approximately constant in the corona discharge for discharge current, and it gradually decreases; the system is stabilized in the arc discharge with the increase in discharge current. The correlation dimension is approximately 1.5 in the corona discharge and 1.35 in the arc discharge, for discharge current. This result indicates that the complexity in the time series has a self-similarity in common with the corona and arc discharge, and the time series in

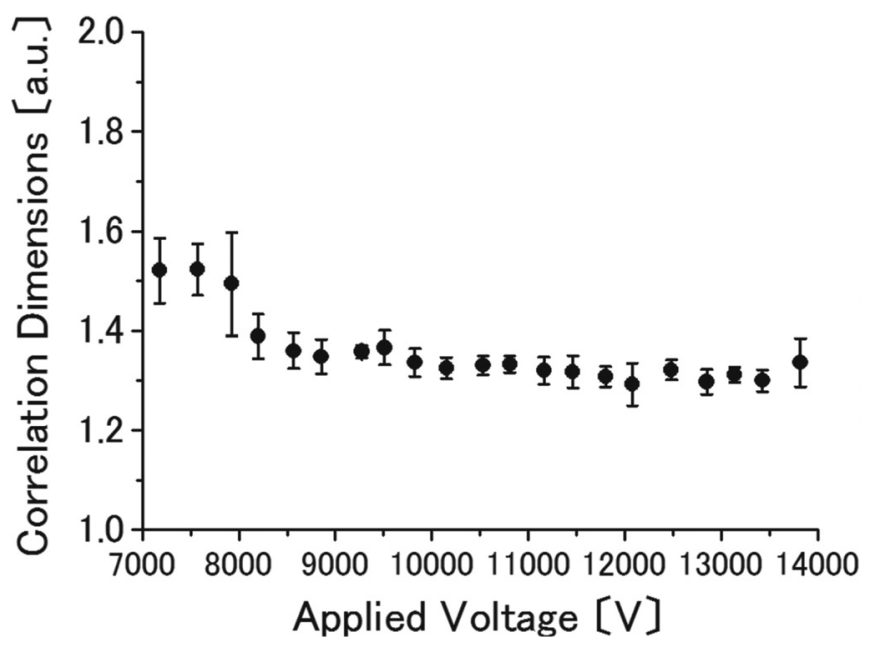

FIG. 8. Graph of the correlation dimensions for discharge voltage. The error bars of each plot have been calculated using the standard deviation of multiple measurements. 
(a)

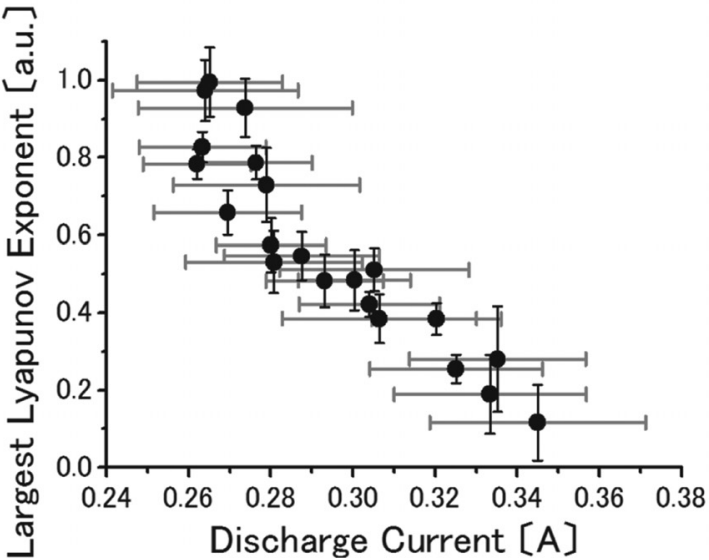

(b)

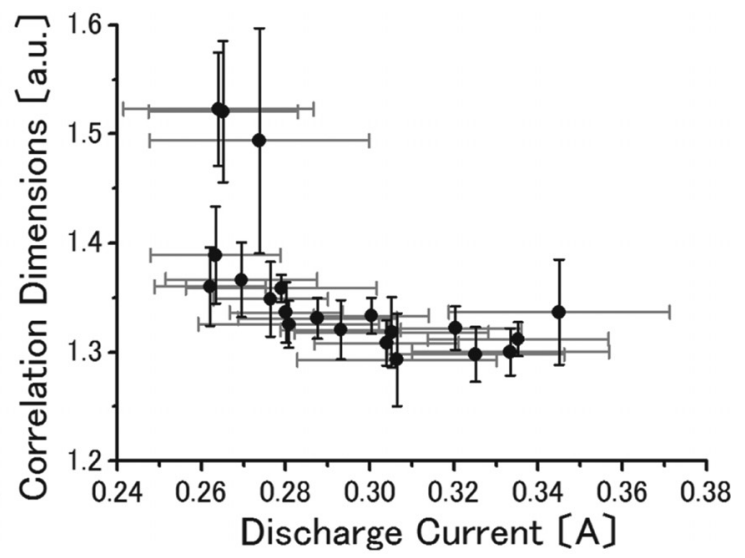

FIG. 9. Graph of the (a) largest Lyapunov exponents and (b) correlation dimensions, for the average intensity of discharge current, obtained in Fig. 4(b). The error bars of each plot have been calculated using the standard deviation of multiple measurements.

the corona discharge has a more complicated structure than that in the arc discharge.

\section{CONCLUSIONS}

In this study, the dynamic behaviors in the time series of a discharge current, in the cases of corona and arc discharge, at atmospheric pressure were investigated from the viewpoints of chaos and fractals. The onset of corona discharge occurs with the insulation breakdown of air when the electric field between two electrodes is beyond a threshold. After the onset of corona discharge, with the further increase in discharge current, the corona discharge gradually changes to an arc discharge. The discharge current for the applied voltage increases in almost direct proportion to the applied voltage between the electrodes.

From the results of the calculation of the largest Lyapunov exponents using the time series sampled in the discharge, it is discovered that the corona discharge is more chaotic than the arc discharge, i.e., the discharge system is stabilized according to the change from corona to arc with the increase in discharge current. From the results of the calculation of the correlation dimension using the time series sampled in the discharge system, it is discovered that it saturates at a non-integer value at every discharge current, i.e., the time series in common with the corona and arc discharge exhibits a self-similarity (fractal), and the corona discharge has a more complicated structure compared to the arc discharge.

During this study on dynamic behaviors of discharge at atmospheric pressure from the viewpoint of both chaos and fractals, a series of experiments were performed under the limited condition of discharge under ac and atmospheric pressure conditions. Changing the frequency of the applied electric field, including dc voltage, and performing experiments under various pressures are challenges for the future. As potential applications from the results of the study, controlling dynamics of corona and arc discharge is expected. The authors express gratitude to Dr. K. Fukushima
(Kumamoto University), Mr. T. Okuda (Ehime University), and the reviewers for their fruitful discussions.

${ }^{1}$ M. Karasik and S. J. Zweben, Phys. Plasmas 7, 4326 (2000).

${ }^{2}$ R. B. Wilson IV and N. K. Podder, Phys. Rev. E 76, 046405 (2007).

${ }^{3}$ S. Lahiri, D. Roychowdhury, and A. N. Sekar Iyengar, Phys. Plasmas 19 082313 (2012).

${ }^{4}$ A. M. Wharton, A. N. Sekar Iyengar, and M. S. Janaki, Phys. Plasmas 20, 022301 (2013)

${ }^{5}$ M. K. Paul, P. K. Sharma, A. Thakur, S. V. Kulkarni, and D. Bora, Phys. Plasmas 21, 062112 (2014).

${ }^{6}$ A. Allagui, A. E. Rojas, T. Bonny, A. S. Elwakil, and M. A. Abdelkareem, J. Appl. Phys. 119, 203303 (2016).

${ }^{7}$ S. W. Teitsworth, R. M. Westervelt, and E. E. Haller, Phys. Rev. Lett. 51, 825 (1983).

${ }^{8}$ R. W. Boswell, Plasma Phys. Controlled Fusion 27, 405 (1985).

${ }^{9}$ P. Y. Cheung, S. Donovan, and A. Y. Wong, Phys. Rev. Lett. 61, 1360 (1988).

${ }^{10}$ N. Ohno, M. Tanaka, A. Komori, and Y. Kawai, J. Phys. Soc. Jpn. 58, 28 (1989).

${ }^{11}$ T. Braun, J. A. Lisboa, and J. A. C. Gallas, Phys. Rev. Lett. 68, 2770 (1992).

${ }^{12}$ S. J. Dodd, L. A. Dissado, J. V. Champion, and J. M. Alison, Phys. Rev. B 52, R16985(R) (1995).

${ }^{13}$ Md. Nurujjaman, R. Narayanan, and A. N. Sekar Iyengar, Chaos 17, 043121 (2007).

${ }^{14}$ X. Tan, Q. Zhang, X. Wang, F. Sun, W. Zha, and Z. Jia, J. Appl. Phys. 104, 103309 (2008).

${ }^{15}$ M. Agop, P. Nica, O. Niculescu, and D.-G. Dimitriu, J. Phys. Soc. Jpn. 81, 064502 (2012).

${ }^{16}$ A. E. Hramov, A. A. Koronovskii, V. A. Maximenko, and O. I. Moskalenko, Phys. Plasmas 19, 082302 (2012).

${ }^{17}$ J. Zhang, Y. H. Wang, and D. Z. Wang, Phys. Plasmas 20, 082315 (2013).

${ }^{18}$ D. Mansuroglu, I. U. Uzun-Kaymak, and I. Rafatov, Phys. Plasmas 24, 053503 (2017)

${ }^{19}$ O. I. Moskalenko, N. S. Frolov, A. A. Koronovskii, and A. E. Hramov, Chaos 27, 126701 (2017).

${ }^{20}$ Th. Pierre, G. Bonhomme, and A. Atipo, Phys. Rev. Lett. 76, 2290 (1996).

${ }^{21}$ K. Taniguchi and Y. Kawai, Phys. Rev. Lett. 83, 548 (1999).

${ }^{22}$ T. Fukuyama, H. Shirahama, and Y. Kawai, Phys. Plasmas 9, 4525 (2002).

${ }^{23}$ P. V. Popov, R. A. Filatov, A. A. Koronovskii, and A. E. Hramov, Tech. Phys. Lett. 31, 221 (2005).

${ }^{24}$ T. Fukuyama, R. Kozakov, H. Testrich, and C. Wilke, Phys. Rev. Lett. 96, 024101 (2006).

${ }^{25}$ N. Chaubey, S. Mukherjee, A. N. Sekar Iyengar, and A. Sen, Phys. Plasmas 22, 022312 (2015).

${ }^{26}$ N. Chaubey, S. Mukherjee, A. Sen, and A. N. Sekar Iyengar, Phys. Rev. E 94, 061201(R) (2016).

${ }^{27}$ T. Fukuyama and M. Okugawa, Phys. Plasmas 24, 032302 (2017). 
${ }^{28}$ T. Fukuyama, K. Hagimine, and R. Miyazaki, J. Phys. Soc. Jpn. 86, 095003 (2017).

${ }^{29}$ L. Niemeyer, L. Pietronero, and H. J. Wiesmann, Phys. Rev. Lett. 52, 1033 (1984).

${ }^{30}$ M. Murat, Phys. Rev. B 32, 8420 (1985).

${ }^{31}$ T. Ficker, J. Appl. Phys. 78, 5289 (1995).

${ }^{32}$ D. I. Iudin, V. Y. Trakhtengerts, and M. Hayakawa, Phys. Rev. E 68, 016601 (2003).

${ }^{33}$ S. Gurlui, M. Agop, M. Strat, G. Strat, S. Bacaita, and A. Cerepaniuc, Phys. Plasmas 13, 063503 (2006).

${ }^{34}$ P. L. Dammig Quiña, I. M. Irurzun, L. M. Salvatierra, and E. E. Mola, Phys. Rev. E 82, 041106 (2010).

${ }^{35}$ M. Megalingam, N. H. Prakash, I. Solomon, A. Sarma, and B. Sarma, Phys. Plasmas 24, 042304 (2017).

${ }^{36}$ A. Wolf, J. B. Swift, H. L. Swinney, and J. A. Vastano, Physica D 16, 285 (1985).

${ }^{37}$ P. Grassberger and I. Procaccia, Physica D 9, 189 (1983).

${ }^{38}$ A. F. Kip, Phys. Rev. 55, 549 (1939).

${ }^{39}$ L. B. Loeb and A. F. Kip, J. Appl. Phys. 10, 142 (1939).

${ }^{40}$ L. B. Loeb, A. F. Kip, G. G. Hudson, and W. H. Bennett, Phys. Rev. 60, 714 (1941).
${ }^{41}$ R. Morrow, Phys. Rev. A 32, 1799 (1985).

${ }^{42}$ M. S. Benilov, Phys. Rev. E 48, 506 (1993).

${ }^{43}$ S. Anders, A. Anders, and I. Brown, Rev. Sci. Instrum. 65, 1253 (1994).

${ }^{44}$ J. Rossignol, S. Clain, and M. Abbaoui, J. Phys. D: Appl. Phys. 36, 1495 (2003).

${ }^{45}$ W. X. Ding, H. Deutsch, A. Dinklage, and C. Wilke, Phys. Rev. E 55, 3769 (1997).

${ }^{46}$ D. Staack, B. Farouk, A. Gutsol, and A. Fridman, Plasma Sources Sci. Technol. 14, 700 (2005).

${ }^{47}$ D. Braun, V. Gibalov, and G. Pietsch, Plasma Sources Sci. Technol. 1, 166 (1992).

${ }^{48}$ M. Klein, N. Miller, and M. Walhout, Phys. Rev. E 64, 026402 (2001).

${ }^{49}$ K. V. Kozlov, H.-E. Wagner, R. Brandenburg, and P. Michel, J. Phys. D: Appl. Phys. 34, 3164 (2001).

${ }^{50}$ J. Ráhel and D. M. Sherman, J. Phys. D: Appl. Phys. 38, 547 (2005).

${ }^{51}$ J. Pons, E. Moreau, and G. Touchard, J. Phys. D: Appl. Phys. 38, 3635 (2005).

${ }^{52}$ Y. Hatta, Gas Discharge (Kindai Kagaku-sha, Tokyo, 1968) (in Japanese).

${ }^{53}$ J. W. Kirchner, Phys. Rev. E 71, 066110 (2005). 